\title{
A Method for Grasp Evaluation Based on Disturbance Force Rejection
}

\author{
Morten Strandberg and Bo Wahlberg, Senior Member, IEEE
}

\begin{abstract}
This paper presents a method for grasp evaluation. It is based on the ability of the grasp to reject disturbance forces. The procedure takes the geometry of object into account, and it is also possible to incorporate task-oriented information. The evaluation criterion is formulated as a min-max optimization problem, for which an efficient algorithm is proposed and analyzed. The result of this algorithm is independent of scale and choice of reference frame, and can easily be visualized as a surface in the force space. The method is illustrated with several examples.
\end{abstract}

Index Terms-Grasp stability, grasping, planning, robot hands.

\section{INTRODUCTION}

$\mathbf{R}$ OBOTS that autonomously grasp objects and interact with them must have methods for choosing appropriate grasps. However, finding a good grasp requires methods for evaluating and comparing different grasps. Furthermore, evaluation can be performed with respect to different properties of the grasp. A most important property of a grasp is its ability to apply forces to the grasped object. A good grasp should also be able to efficiently reject external disturbance forces, especially those forces that are expected to occur during the task that is to be performed. Grasps should also be robust to errors in models and sensor data.

Robotic grasping and manipulation is an extensive research area. A survey of the state-of-art up to around 2001 is presented in, e.g., [1] and [2]. An in-depth treatment of robotic grasping is given in [3] and [4]. As discussed in, e.g., [5], automatic grasp planning for robot manipulators is a difficult problem due to its high degree of freedom. Computationally efficient methods for grasp evaluation are of fundamental importance when deriving useful grasp-planning algorithms.

In this paper, we present a framework for grasp evaluation that is based on the grasp's ability to resist disturbance forces. The approach leads to a min-max formulation, for which we propose an efficient algorithm. The result of the algorithm is easily visualized as a surface in the force space. For polyhedral objects, we give a proof showing that only the vertices of the

Manuscript received March 17, 2005. This paper was recommended for publication by Associate Editor D. Prattichizzo and Editor H. Arai upon evaluation of the reviewers' comments. This work was supported by the Swedish Foundation for Strategic Research through the Centre for Autonomous Systems. This paper was presented in part at the IEEE/RSJ International Conference on Intelligent Robots and Systems, Lausanne, Switzerland, 2002.

M. Strandberg was with the Automatic Control Laboratory and Centre for Autonomous Systems, Royal Institute of Technology (KTH), SE-100 44 Stockholm, Sweden. He is now with ABB Corporate Research, SE 72178 Västerås, Sweden (e-mail: Morten.Strandberg@se.abb.com).

B. Wahlberg is with the Automatic Control Laboratory and Centre for Autonomous Systems, Royal Institute of Technology (KTH), SE-100 44 Stockholm, Sweden (e-mail: bo.wahlberg@s3.kth.se).

Digital Object Identifier 10.1109/TRO.2006.870665 object need to be considered. Compared with other approaches to grasp evaluation, the main benefits are as follows.

- The procedure incorporates the complete object geometry.

- Task information is easily included, and actually reduces the computational complexity.

- The result is independent of scale and choice of reference frame.

- The result can be visualized in 3-D and is easy to interpret.

The next section gives a brief introduction to grasp analysis. In Section III, we discuss related work, and in Section IV, the proposed grasp-evaluation procedure is presented. To demonstrate the procedure, we give some examples in Section V. The paper ends with a summary.

\section{GRASP ANALYSIS}

In this section, we give a brief introduction to grasp analysis and introduce the notation and concepts that are used in the context of grasp evaluation.

It is assumed that the grasped object is rigid, and that the grasp consists of any number of point contacts with friction. The point-contact assumption might seem limiting but, as was pointed out by Nguyen [6], any planar polygonal contact can be represented as the convex sum of point contacts placed at the vertices of the contact polygon. Attached to the object is a reference frame, to which all contacts and forces are related.

Each contact will have its own reference frame, with the $z$-axis pointing in the direction of the inward surface normal, see Fig. 1(a). Because of friction being present, the contact force can deviate from the $z$-axis. If the contact forces obey the Coulomb friction model, then the space of all admissible contact forces forms a circular cone with opening angle $2 \tan ^{-1}(\mu)$, where $\mu$ is the coefficient of friction. This cone, called the friction cone, will impose nonlinear constraints on the contact force components.

In the literature, the circular friction cone is often approximated with an $n$-sided pyramid, see Fig. 1(b). By doing this, we can write the contact force as a positive linear combination of the force vectors spanning the pyramid

$$
\mathbf{f}=\sum_{j=1}^{n} \alpha_{j} \mathbf{f}_{j}, \quad \alpha_{j} \geq 0 .
$$

Note that by choosing the vectors $\mathbf{f}_{1}, \ldots, \mathbf{f}_{n}$ to have a unit $z$-component, the normal component of the contact force is easily obtained as $\sum_{j=1}^{n} \alpha_{j}$.

It is often convenient to concatenate force and torque vectors $\mathbf{F}$ and $\mathbf{T}$ into a wrench, defined as $\mathbf{W}=\left(\mathbf{F}^{\mathrm{T}}, \mathbf{T}^{\mathrm{T}}\right)^{\mathrm{T}}$. A wrench is thus a 6-D column vector. 


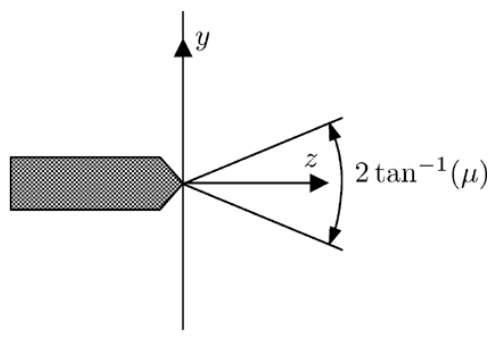

(a) Point contact

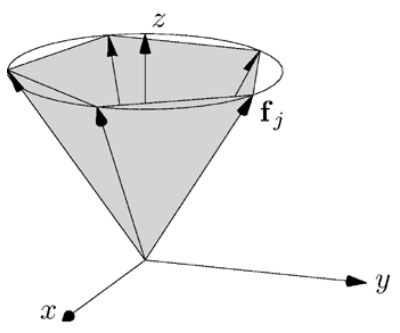

(b) Friction cone approximation

Fig. 1. For nonslipping contacts that obey the Coulomb friction model, the contact forces must be inside the friction cone. (a) Side view of a point contact, together with its coordinate system. (b) Example of a friction cone approximated by a five-sided pyramid.

Each force $\mathbf{f}_{j}$ will result in an object wrench $\mathbf{w}_{j}$, which can be computed if the position and the orientation of the contact relative to the object frame is known. Let the $\mathbf{w}_{j}$ from all contacts be the columns of a $6 \times m n$ matrix $\mathbf{G}$, where $m$ is the number of contacts. This matrix is called the grasp matrix. Summing up the contributions from all contacts, the total wrench exerted by the grasp on the object, $\mathbf{W}$, can be written as

$$
\mathbf{W}=\mathbf{G x}, \quad x_{k} \geq 0, \quad k=1, \ldots, m n
$$

where $\mathbf{x}$ is a vector containing the $\alpha_{j}$ for all contacts. See [4] for more details on how to construct the grasp matrix. When analyzing a grasp, it is of interest to know the space of wrenches that can be applied to the object by the grasp. The unit grasp wrench space (UGWS) is often ${ }^{1}$ defined as the space of wrenches that satisfies (2) and $\sum_{k=1}^{m n} x_{k}=1$. This space is equal to the convex hull of $\mathbf{G}$, which can be efficiently computed using the Quickhull algorithm, see [8].

An important class of grasps are those that have force closure. A grasp has force closure if there exists a solution to (2) for any $\mathbf{W}$. This means that the grasp can counteract any external wrench acting on the body by adjusting the contact forces properly. If a grasp has force closure, then the convex hull of $\mathbf{G}$ must contain a neighborhood of the origin [4]. The converse is also true: if the convex hull contains a neighborhood of the origin, then the grasp has force closure.

\footnotetext{
${ }^{1}$ Note that by choosing another norm for the contact force vector, we get other definitions for the UGWS. Another common, and more natural, definition of the UGWS is to limit the normal component of each individual contact to one, see [7]. With this definition, however, the UGWS is much more costly to compute, and therefore, the definition based on the sum of all contact forces is more common.
}

\section{RELATED WORK}

Grasp evaluation can be performed with respect to different properties of the grasp. Which properties are important is determined by the context and the task. For example, when inserting a peg into a hole, it is important that the grasp is compliant, to not give rise to large contact forces due to position and orientation errors. For such a task, it is important that the grasp is not only stable, but also that it has a high manipulability measure. Grasp manipulability is the degree to which the fingers can impart arbitrary motions to the object, see [9].

Grasp Robustness: Considering the uncertainties in models and sensor data, grasps that are robust to positioning and modeling errors are to be preferred. Nguyen [6] addressed this by developing algorithms for finding maximal independent contact regions for several important types of grasps; among them, two-finger grasps of polygons. As long as the contacts are within these regions, the grasp has force closure. Ponce and Faverjon [10] extended the results of Nguyen to three-finger grasps of polygons. Thus, if robustness to position and modeling errors are of concern, a grasp quality measure could be chosen as the length of the smallest contact region.

Bone and $\mathrm{Du}$ [11] derived a measure of robustness to positional errors for polygon grasps. They consider all combinations of finger displacements and evaluate the maximum torque magnitude the resulting grasp can resist. The robustness measure is the sum of the relative change in torque-resisting capability for all the displacements.

It is well known that friction helps to make a grasp more stable. In situations where the coefficient of friction is not known beforehand, we should favor grasps whose stability is not dependent on a large coefficient of friction. Mantriota [12] defined as quality measure the minimum coefficient of friction needed to resist a set of unit disturbance wrenches, directed along the principal wrench-space directions. The resulting grasps are robust in the sense that they rely as little as possible on friction.

Wrench-Space Volumes: Another important property of a grasp is its ability to apply forces to the grasped object. A good grasp should be able to efficiently counteract external forces, especially those forces that are expected to occur during the task at hand. Previous work focusing on this aspect of a grasp is naturally divided into task-independent quality measures and task-directed quality measures. In task-independent grasp evaluation, information about the task is assumed to be unavailable or ignored, and the resulting grasp quality measure therefore reflects, in some way, the overall stability of the grasp. In task-directed grasp evaluation, on the other hand, the suitability of a grasp with respect to a particular task is evaluated. Both types of quality measures are often based on the UGWS. The wrench space is 6-D and, intuitively, a good grasp should have a large UGWS whose shape matches the set of wrenches that are expected to occur for the given task (the task wrench space).

Kirkpatrick et al. [13] proposed a quality measure which is the radius of the largest wrench-space ball that just fits within the UGWS. This measure was also used in [7] and [14]. This quality measure is task-independent, and it assumes that all directions in the wrench space are equally important. There are two drawbacks with this kind of measure: it is not scale-invariant; and it is 


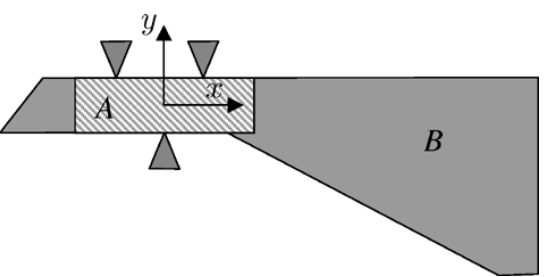

Fig. 2. Two grasps of two different geometries. One grasp is drawn on top of the other to emphasize that the placement of the contacts are identical. The stability of the grasp will depend on which object is grasped, Object $A$ or Object $B$. Most grasp quality measures do, however, only use the contact information.

not invariant to translations of the torque origin. The first drawback is easily remedied by normalizing the torque components of the wrench vector, e.g., with the inverse of the maximum distance from the torque origin to the surface of the object [14]. The second drawback is more severe, because clearly, a grasp quality measure should not depend on the choice of reference frame. This issue was addressed in [15], which proposed as an invariant measure the radius of the largest wrench-space ball with respect to all possible coordinate frames. The approach does, however, lack a simple physical interpretation: the choice of coordinate frame for evaluating a grasp is dependent on the grasp itself. A much simpler invariant measure was proposed by Li and Sastry [16]; they suggested using the volume of the UGWS as an invariant measure. Note, however, that a volume-based quality measure can give a nonzero quality to grasps that do not have force closure, i.e., are unable to resist forces in some directions.

Many of the problems with quality measures that are based on wrench-space balls arise because we are trying to compare forces with torques, which does not make sense, as they have different units. To avoid such comparisons, [17] used a decoupled approach, leading to a two-valued quality measure. The optimal grasp is the one that lexicographically maximizes both values; they first compute the grasps that best counteract pure forces, and then select from among those grasps the one that best resists pure torques.

To obtain task-directed measures, [16] suggested the use of 6-D task ellipsoids, whose shape resembles the space of forces and torques encountered in the task. It defined a task-directed quality measure as the largest scale factor that causes the task ellipsoid to be embedded in the UGWS. However, as pointed out in [16], "The process of modeling a task by a task ellipsoid is quite complicated."

Including the Object Geometry: Using the UGWS alone for the construction of a quality measure has a drawback that, seemingly, has not received much attention: the wrench space is constructed from information about the contacts alone. Hence, the effects of the complete object geometry on grasp stability are not taken into account. A grasp quality measure that does not take object geometry into account would treat the two grasps in Fig. 2 as equal; intuition, however, tells us that the grasp of Object $A$ should be more stable than that of Object $B$.

Introducing the object wrench space (OWS) concept, [14] actually incorporates the complete object geometry into the grasp evaluation. The OWS represents the best grasp of the object that can ever be achieved. An alternative interpretation is that the OWS is the set of wrenches that can be created by a (unit) dis- tribution of disturbance forces acting anywhere on the surface of the object. Thus, the OWS depends on the geometry of the object. The OWS concept is closely related to the idea presented herein; however, the examples presented in [14] on this subject are limited to 2-D polygons with frictionless point contacts.

In a recent approach, [18] combined the OWS concept of [14] with the task ellipsoids of [16]. If no task information is given, the best assumption one can make about the disturbance wrenches is that they are distributed according to the OWS. Borst et al. [18] therefore choose as task ellipsoid an ellipsoid that tightly encloses the OWS. As the task ellipsoid is automatically constructed, it removes one of the strongest objections against using them. It is also worth noting that their method for computing the grasp quality does not rely on any friction cone approximations.

Visualization: Visualization of a 6-D wrench space is impossible, and therefore, many papers only dealt with 2-D grasping problems. The wrench space of a 2-D grasping problem is 3-D and easy to visualize. Miller and Allen [19] suggested several methods for projecting the 6-D wrench space into 3-D. Using these projections, important characteristics of the 6-D wrench space could be visualized. The approach presented here can be seen as a natural projection from the 6-D wrench space to the 3-D force space.

Compliant Grasps: In the work presented so far, grasp compliance has not been addressed. This is also the case of the method proposed in this paper. For compliant grasps, the grasp stiffness matrix is a useful tool, see, e.g., [20]. A compliant grasp is stable if the grasp stiffness matrix is positive definite. It seems natural to base a quantitative measure of stability on the eigenvalues of the stiffness matrix, but care must be taken, as these are not invariant under a change of reference frame; see, e.g., [21] and [22].

Bruyninckx et al. [22] derived a grasp quality measure based on the generalized eigenvalue decomposition of the grasp stiffness matrix. The generalization requires a choice of a metric on the group of rigid-body displacements that enables the identification of twists with wrenches. Lin et al. [21] derived a frameinvariant quality measure in terms of the principal translational and rotational stiffness parameters. By introducing a physically based conversion of rotational stiffness parameters into equivalent translational stiffness, [21] overcame the problem of comparing translational and rotational stiffness parameters.

\section{GRasp-Evaluation Procedure}

As was pointed out in [16], modeling a task using 6-D task ellipsoids is laborious. Furthermore, visualization of the wrench space is impossible, unless the grasp problem is 2-D. We argue that in many cases, the explicit use of the torque component is not necessary, thereby reducing the 6-D wrench space to a 3-D force space.

Key Idea: Consider how disturbance wrenches are applied to a grasped object: in almost all practical cases, a disturbance wrench arises from a pure force acting on the surface of the object. The resulting torque component is immediately given by

$$
\mathbf{T}=\mathbf{a} \times \mathbf{F}
$$


where $\mathbf{a}$ is a vector specifying where the force $\mathbf{F}$ is applied. Specifying the grasp matrix $\mathbf{G}$ and $\mathbf{a}$ in the same coordinate system is key here. Using the same torque origin assures that the result will be independent of the choice of frame. According to (3), the torque component is not independent of the applied force, and they are always orthogonal to each other. Based on this observation, the grasp-evaluation procedure described below is proposed.

Consider a unit vector ê, representing a fixed direction for the disturbance force so that the disturbance force can be written as $f \hat{\mathbf{e}}$, where $f$ is a dimensionless scalar. Sweep this disturbance force over the surface of the object, finding the smallest, positive $f$ that results in a wrench that is exactly on the border of the UGWS. Let us denote this value by $f^{\star}$. Note that when performing this "sweeping" operation, only those points on the object for which $\hat{e}$ is inside the friction cone can come into consideration. We also require $f \geq 0$, because we cannot allow tractional disturbance forces. Repeating this process for all directions of the disturbance force, we will end up with a closed surface $S$ in force space. Specifying force directions with spherical coordinates $\varphi$ and $\theta$, the surface $S$ is given by the vectors $\left\{f_{\varphi \theta}^{\star} \hat{\mathbf{e}}_{\varphi \theta}\right\}$. The interpretation of this surface is straightforward. If a disturbance force is inside $S$, a unit grasp will be able to resist the resulting wrench, no matter where the force is applied.

Min-Max Formulation: The above procedure can be formulated mathematically as a min-max problem. Let $\partial D$ denote the surface of the object and $\mathrm{FC}_{\mathbf{a}}$ the friction cone at the surface point specified by the vector $\mathbf{a}$. To include forces that cannot be seen as a force acting on the surface of the object (e.g., gravitational forces), we can include an offset wrench $\mathbf{W}_{0}$. For each direction ê, we want to solve the following problem:

$$
\begin{array}{r}
f^{\star}=\min _{\mathbf{a}} \max _{\mathbf{x}}\left\{f \in \mathbb{R}^{+}: \quad-\mathbf{W}_{0}-f\left[\begin{array}{c}
\hat{\mathbf{e}} \\
\mathbf{a} \times \hat{\mathbf{e}}
\end{array}\right]=\mathbf{G x},\right. \\
\left.\hat{\mathbf{e}} \in \mathrm{FC}_{\mathbf{a}}, \quad \mathbf{a} \in \partial D, \quad x_{i} \geq 0, \quad \sum_{i=1}^{m n} x_{i}=1\right\} .
\end{array}
$$

The resulting surface $S$ can be seen as the space of admissible disturbance forces around $\mathbf{W}_{0}$, assuming a unit grasp and that the disturbance forces are always applied at the worst-case surface point. Taking the complete geometry into account, we thereby assume that disturbance forces can occur anywhere on the object surface. However, as illustrated by the following example, task information can easily be included by only choosing those parts of the object surface where disturbance forces are likely to occur.

Task-Directed Evaluation: The usual way to hold a pen when writing is to place the fingertips close to the tip of the pen, letting its upper part rest between the thumb and the index finger, see Fig. 3. Clearly, this grasp cannot easily resist forces acting on the upper part of the pen. Using the complete surface of the pen as input to the evaluation procedure would thus result in a poor overall stability for the grasp. However, when writing, we know that external forces are only exerted on the tip of the pen, and the chosen grasp is excellent for balancing those forces. Accordingly, a task-directed measure, indicating a more stable grasp, can be computed by using only the tip of the pen as input.

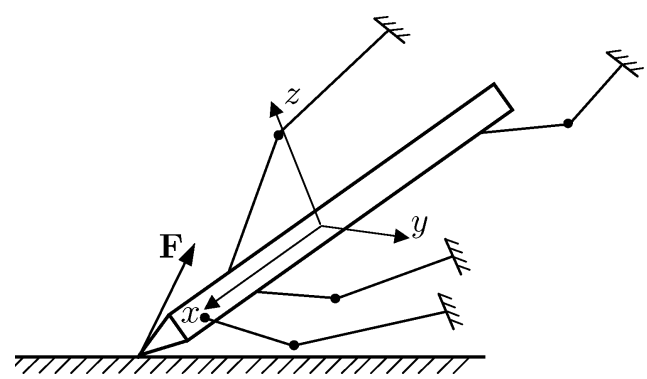

Fig. 3. The above grasp for holding the pen is good at resisting forces that are applied to the tip of the pen, but bad at resisting forces at the upper part. A task-directed evaluation of the grasp would only include only the tip of the pen, as this is where external forces are expected to occur.

Another way to add task information is through $\mathbf{W}_{0}$. If the object is held in the same orientation during the task, then we can easily include the effect of gravity with $\mathbf{W}_{0}$. In some cases, this wrench can actually help to stabilize the grasp, e.g., when carrying a plate resting on the open palm.

Disturbance-Force Friction Cone: Note that upon using only parts of the object surface, $S$ might not be closed. This can happen if some of the force directions ê never fall inside a friction cone. The same can happen if the assumed friction cone for the disturbance force is very small. However, choosing large enough friction cones for the disturbance forces, the procedure will cover the force directions relevant to the task. Thus, the procedure depends on some imagined coefficient of friction, $\hat{\mu}$, that should be chosen considerably larger than the real one.

Remark: For $\hat{\mu}_{1}>\hat{\mu}_{2}$, we will have $f_{1}^{\star} \leq f_{2}^{\star}$. Thus, increasing $\hat{\mu}$ will always result in a more conservative evaluation of the grasp.

Disturbance-Force Upper Bound: Since we assume a unit grasp, there exists a theoretical upper bound for $S$. It is easily shown that if $\mathbf{W}_{0}=0$, then $S$ is bounded by a sphere with radius $\sqrt{1+\mu^{2}}$. For a nonzero offset wrench, this bounding sphere will be distorted and translated.

\section{A. Algorithm for Solving the Min-Max Problem}

One immediate objection against the proposed procedure is that it seems both unnecessary and computationally overwhelming to traverse the entire surface of the object. It is, however, shown in Section IV-C that if the object is a polyhedron, then it is enough to traverse its vertices only. Therefore, in the following analysis, we will assume polyhedral objects.

The UGWS can be seen as the interior of a set of $N_{\mathrm{P}}$ hyperplanes in 6-D. Each hyperplane has a unit normal $\mathbf{n}_{p}$ and an offset $d_{p}^{\prime}$. The purpose of the prime will be explained below. For a wrench $\mathbf{W}$ to be inside the hull, we must have

$$
\mathbf{n}_{p}^{\mathrm{T}} \mathbf{W}+d_{p}^{\prime} \leq 0, \quad p=1, \ldots, N_{\mathrm{P}}
$$

Looking for wrenches that are exactly on the boundary of the wrench space could be done by looking for intersections with these hyperplanes. Here, we use the Quickhull program [8] to compute the hyperplanes. 
Clearly, we must discretize the space disturbance force directions. This is most easily done using spherical coordinates $\hat{\mathbf{e}}=(\cos \varphi \sin \theta, \sin \varphi \sin \theta, \cos \theta)^{\mathrm{T}}$, where $\theta \in[0, \pi]$ and $\varphi \in[0,2 \pi)$. Assume that the grasp must resist some default offset wrench $\mathbf{W}_{0}$. If, in addition, a disturbance force is applied at a point $\mathbf{a}$ on the object's surface, then the following must hold for the grasp to remain stable:

$$
-\mathbf{W}_{0}-f\left[\begin{array}{c}
\hat{\mathbf{e}} \\
\mathbf{a} \times \hat{\mathbf{e}}
\end{array}\right]=\mathbf{G x}, \quad x_{i} \geq 0, \quad \sum_{i=1}^{m n} x_{i}=1 .
$$

The minus signs in the left-hand side (LHS) of (6) are necessary, because the grasp has to exert a wrench that cancels the external wrench to maintain equilibrium.

With ê and a constant, the LHS of (6) describes a line in wrench space parameterized by $f$

$$
\mathbf{W}=-\mathbf{W}_{0}-f \mathbf{W}^{\star}
$$

where we have introduced the convenient notation

$$
\mathbf{W}^{\star}=\left[\begin{array}{c}
\hat{\mathbf{e}} \\
\mathbf{a} \times \hat{\mathbf{e}}
\end{array}\right]
$$

Thus, if $-\mathbf{W}_{0}$ is inside the hull, there exist two intersections with the hull boundary and the line: one with negative $f$, which we are not interested in, and one with positive $f$, which we are looking for. Now we only need an effective algorithm for finding this positive $f$-value for each force direction.

Assume that the wrench $\mathbf{W}$ from (7) is exactly on hyperplane $p$. Then from (5), we obtain

$$
-\mathbf{n}_{p}^{\mathrm{T}}\left(\mathbf{W}_{0}+f \mathbf{W}^{\star}\right)+d_{p}^{\prime}=0, \quad p=1, \ldots, N_{\mathrm{P}} .
$$

We note that the term $-\mathbf{n}_{p}^{\mathrm{T}} \mathbf{W}_{0}$ can be seen as coordinate translation of the wrench space, changing the plane offsets. Thus, we can introduce $d_{p}=d_{p}^{\prime}-\mathbf{n}_{p}^{\mathrm{T}} \mathbf{W}_{0}$, which simplifies (9) to

$$
-f \mathbf{n}_{p}^{\mathrm{T}} \mathbf{W}^{\star}+d_{p}=0, \quad p=1, \ldots, N_{\mathrm{P}} .
$$

Since it is assumed that the grasp is able to resist the offset wrench, we must have $d_{p} \leq 0$ for all hyperplanes. This is a more general condition than the commonly used $d_{p}^{\prime}<0$, which requires the grasp to have force closure. With only one unknown in (10), we can solve directly for $f$

$$
f_{p}=\frac{d_{p}}{\mathbf{n}_{p}^{\mathrm{T}} \mathbf{W}^{\star}}, \quad p=1, \ldots, N_{\mathrm{P}}
$$

The smallest positive $f_{p}$, taken over all hyperplanes, will correspond to the largest disturbance force, with direction $\hat{e}$, that can be applied to a particular vertex a. Repeating this procedure for all vertices, for which ê is inside the friction cone, will give the worst-case disturbance force. However, computing $f_{p}$ according to (11) for all hyperplanes and all vertices seems highly inefficient. The complexity of such an algorithm would be close to $O\left(N_{\mathrm{V}} N_{\mathrm{P}}\right)$, where $N_{\mathrm{V}}$ is the number of vertices on the object. In reality, it would be slightly lower, due to ê falling outside the friction cone for many vertices.

\section{B. Algorithm Improvement}

One way of reducing the complexity would be to precompute and keep track of the minimum, positive $f$ that can be achieved from each hyperplane. Calling these minimum values $f_{\min , p}$, we can sort the hyperplanes so that $f_{\min , p}$ is always increasing. This way, we are likely to find the limiting hyperplane earlier, and we can stop the loop as soon as the current minimum $f$ is smaller than $f_{\min , p}$. Using (8) and (11) together with $\|\hat{\mathbf{e}}\|=1$, we find the following simple lower bound:

$$
f_{\min , p}=\frac{-d_{p}}{\sqrt{n_{1 p}^{2}+n_{2 p}^{2}+n_{3 p}^{2}}+a_{\max } \sqrt{n_{4 p}^{2}+n_{5 p}^{2}+n_{6 p}^{2}}}
$$

where $a_{\max }$ is the maximum vertex distance. Note that the minus sign is necessary, because $d_{p}$ is negative.

Noticing that $\|\mathbf{a} \times \hat{\mathbf{e}}\|$ is constant while looping over the hyperplanes, this sorting idea can be further refined. With knowledge about $\|\mathbf{a} \times \hat{\mathbf{e}}\|$, we can obtain a better lower bound than that of (12). So instead of sorting only once, sorting can be done $N_{B}$ times, where each sorting is associated with an interval for $\|\mathbf{a} \times \hat{\mathbf{e}}\|$. Thus, we have $N_{B}$ buckets, where the hyperplane ordering in each bucket $b$ is determined by sorting them increasing in $f_{\min , p}^{b}$, given by

$$
f_{\min , p}^{b}=\frac{-d_{p}}{\sqrt{n_{1 p}^{2}+n_{2 p}^{2}+n_{3 p}^{2}}+\frac{a_{\max }}{b} \sqrt{n_{4 p}^{2}+n_{5 p}^{2}+n_{6 p}^{2}}} .
$$

So if $(b-1) a_{\max } / N_{B} \leq\|\mathbf{a} \times \hat{\mathbf{e}}\|<b a_{\max } / N_{B}$, the current cross-product belongs to bucket $b$. One could find even better values for $f_{\min , p}^{b}$ using the fact that $\hat{\mathbf{e}} \perp(\mathbf{a} \times \hat{\mathbf{e}})$, but the resulting optimization problem would cost too much to make it worthwhile.

Improvement From Sorting: To study the effect of sorting the hyperplanes, the grasp of Fig. 5(a) is evaluated for varying values of $N_{B}$. The input size is 8 object vertices, 416 hyperplanes, and 578 force directions. The required computational time, for each value of $N_{B}$, was estimated by taking the average of 1000 evaluations. The time for constructing the convex hull using the Quickhull algorithm was not included. The computations were done on a computer with a Pentium M processor running at $1.6 \mathrm{GHz}$.

The results are shown as three curves in Fig. 4, where each curve represents the grasp in Fig. 5(a), but with a different gravitational load. In all cases, we see a significant drop in the computational time as $N_{B}$ increases. For each problem, there is an optimal value of $N_{B}$, beyond which the computational time starts to increase rather slowly. Hence, it is better to overestimate $N_{B}$. 


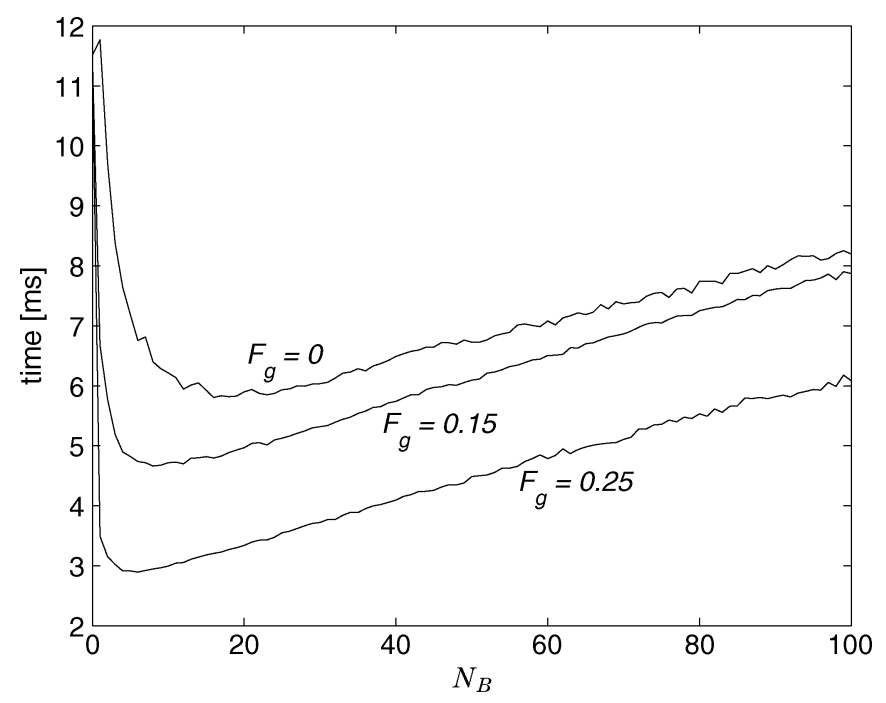

Fig. 4. Grasp from Fig. 5(a) (8 object vertices, 416 hyperplanes, and 578 force directions) was evaluated with varying gravitational load $F_{g}$. The curves show required computational time, in milliseconds, as a function of $N_{B}$. For the special case $N_{B}=0$, no sorting at all is done. Note that the effect from sorting is problem-dependent.

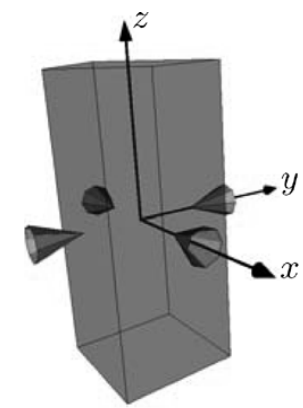

(a)

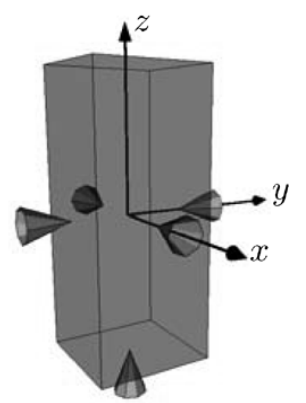

(b)
Fig. 5. Illustration of the grasps evaluated in the examples. The box dimensions are $2 \times 2 \times 5$, and gravity is directed along the negative $z$-axis. The coefficient of friction is set to 0.3 for all the contacts, and each friction cone is approximated by eight force vectors. Note that the friction cones have been plotted on the outside of the object to make them more visible. (a) Four point contacts. (b) Five point contacts.

In the implementation of the hyperplane sorting, a variation of the Radix Sort algorithm [23] was used. ${ }^{2}$ This algorithm is special, in that it has a linear complexity in the number of elements to be sorted. In this case, sorting always requires four traversals over the hyperplanes, thus we know that the time spent on sorting will be exactly proportional to the number of buckets. As the number of buckets are increased, at some point, there will be no further gain from having the hyperplanes sorted. Any further increase in the number of buckets will then cause a linear increase of the total computational time. This is also seen in Fig. 4.

\footnotetext{
${ }^{2}$ In books on algorithms, Radix Sort is described as an algorithm for sorting integer values. It is, however, due to the IEEE Standard 754 floating-point representation, possible to use it for sorting floating-point numbers as well; if they are interpreted as integers, comparisons between them will still be correct (although some care has to be taken with the sign bit).
}

For optimal effect of the sorting, the number of buckets should be set adaptively for each problem, depending upon the number of object vertices and the number of hyperplanes. However, for the curves in Fig. 4, the numbers of vertices and the number of hyperplanes are constant. Thus, the effect from sorting is very problem-dependent, and difficult to predict from the size of the input. The experiments so far have shown that choosing $N_{B}=30$ gives a significant performance increase for most problems.

Assuming that the vertices have a uniform range distribution around the origin, the limiting, or worst-case vertices will often be those which generate large torque components. A further optimization is therefore to sort the vertices, decreasing in $\|\mathbf{a} \times \hat{\mathbf{e}}\|$, before we loop over them. To satisfy the assumption about uniform range distribution, the object frame should be placed at the object's centroid. The final algorithm is described in Algorithm 1.

\section{Algorithm 1 Compute disturbance-force surface}

choose object vertices relevant to the task

give the offset wrench $\rightarrow \mathbf{W}_{0}$

compute the convex hull of $\mathbf{G}, \rightarrow\left\{\mathbf{n}, d^{\prime}\right\}$

transform the hyperplanes, $d_{p}=d_{p}^{\prime}-\mathbf{n}_{p}^{\mathrm{T}} \mathbf{W}_{0}$

if $\left(d_{p}>0\right)$ then

abort $\left\{\mathbf{W}_{0}\right.$ is not inside the hull $\}$

\section{end if}

sort hyperplanes $N_{B}$ times, increasing in $f_{\min , p}^{b}$

for all force directions $\hat{\mathbf{e}}_{i}$ do

select vertices $\mathbf{a}_{j}$ for which $\hat{\mathbf{e}}_{i} \in \mathrm{FC}_{j}$

sort vertices, descending in $\left\|\mathbf{a}_{j} \times \hat{\mathbf{e}}_{i}\right\|$

$f_{i}^{\star}=+\infty$

for all sorted vertices $\mathbf{a}_{j}$ do

find bucket, $b=N_{B}\left\|\mathbf{a}_{j} \times \hat{\mathbf{e}}_{i}\right\| / a_{\max }$

for all sorted hyperplanes $\mathbf{n}_{p}, d_{p}$ do

$$
\begin{aligned}
& \text { if }\left(f_{i}^{\star}<f_{\min , p}^{b}\right) \text { then } \\
& \text { break }
\end{aligned}
$$

end if

$$
\begin{aligned}
& f_{\text {cand }}=d_{p} /\left(\mathbf{n}_{p}^{\mathrm{T}} \mathbf{W}^{\star}\right) \\
& \text { if }\left(f_{\text {cand }} \geq 0 \text { AND } f_{\text {cand }}<f_{i}^{\star}\right) \text { then } \\
& \qquad f_{i}^{\star}=f_{\text {cand }} \\
& \text { end if }
\end{aligned}
$$

\section{end for}

\section{end for}




\section{Disturbance Forces and Object Vertices}

An important question is how many points on the surface of the body we have to consider when moving around the disturbance force. Here we will give a proof showing that, if the body is a polyhedron, then it is sufficient to consider only its vertices.

Theorem IV.1: For a polyhedral body grasped by a unit grasp, the worst point of attack for a disturbance force will always be a vertex of the body.

Proof: Without loss of generality, we assume that each face of the polyhedral body is a convex polygon; in case of a nonconvex polygon, we can always decompose it into a finite number of convex polygons. The $k$ th face is the convex hull of the vertices $\mathbf{a}_{1}, \mathbf{a}_{2}, \ldots, \mathbf{a}_{N_{k}}$, where $N_{k}$ is the number of vertices. ${ }^{3}$ Any position a on the $k$ th face can thus be written as a convex combination of its vertices

$$
\mathbf{a}=\sum_{i=1}^{N_{k}} \beta_{i} \mathbf{a}_{i}, \quad \sum_{i=1}^{N_{k}} \beta_{i}=1, \quad \beta_{i} \geq 0 .
$$

For a given disturbance-force direction ê, we only have to consider the subset of faces for which the disturbance force satisfies the friction cone constraint. In this subset, let us now consider the $k$ th face, so that the position of the disturbance force can be expressed with (14). Using (8), (11), and (14), we can write the intersection between the applied wrench and the $p$ th hyperplane as

$$
f_{p}=\frac{d_{p}}{b_{0 p}+b_{1 p} \beta_{1}+\ldots+b_{i p} \beta_{i}+\ldots+b_{N_{k} p} \beta_{N_{k}}}
$$

where $b_{0 p}=n_{1 p} e_{x}+n_{2 p} e_{y}+n_{3 p} e_{z}$ and $b_{i p}, 1 \leq i \leq N_{k}$ can be computed from $n_{4 p}, n_{5 p}, n_{6 p}, e_{x}, e_{y}, e_{z}$, and $\mathbf{a}_{1}, \ldots, \mathbf{a}_{N_{k}}$. It is seen that for each hyperplane, the minimum positive $f_{p}$ is achieved by letting the $\beta_{i}$ with smallest coefficient become one (note that $d_{p}$ is negative for a stable grasp). This clearly corresponds to one of the vertices of the polygon. So for each hyperplane, the worst point of attack for the disturbance force will correspond to a vertex of the polygon. Hence, the overall worst point of attack will also be a vertex.

Since it is sufficient to consider only the vertices for each polygon face, we conclude that we only have to consider the vertices of a polyhedral body. Note that this conclusion is independent of the friction cone opening angle. Narrowing the friction cone will only reduce the number of polygon faces that has to be considered for each disturbance-force direction.

\section{EXAMPLES}

To illustrate the procedure proposed in this paper, it was tested on two small problems. For both problems, the coefficient of friction was set to 0.3 , and each friction cone was approximated by eight force vectors. The resolution for the disturbance force directions are set to $10^{\circ}$ for both $\varphi$ and $\theta$, resulting in a total of 578 force directions. This simple discretization scheme will

\footnotetext{
${ }^{3} \mathrm{~A}$ more strict notation would show that the vertices of each polygon face is a subset of the vertices of the whole body. However, besides running out of letters, we believe that additional indexes in this case would only lead to a notation that is harder to read.
}
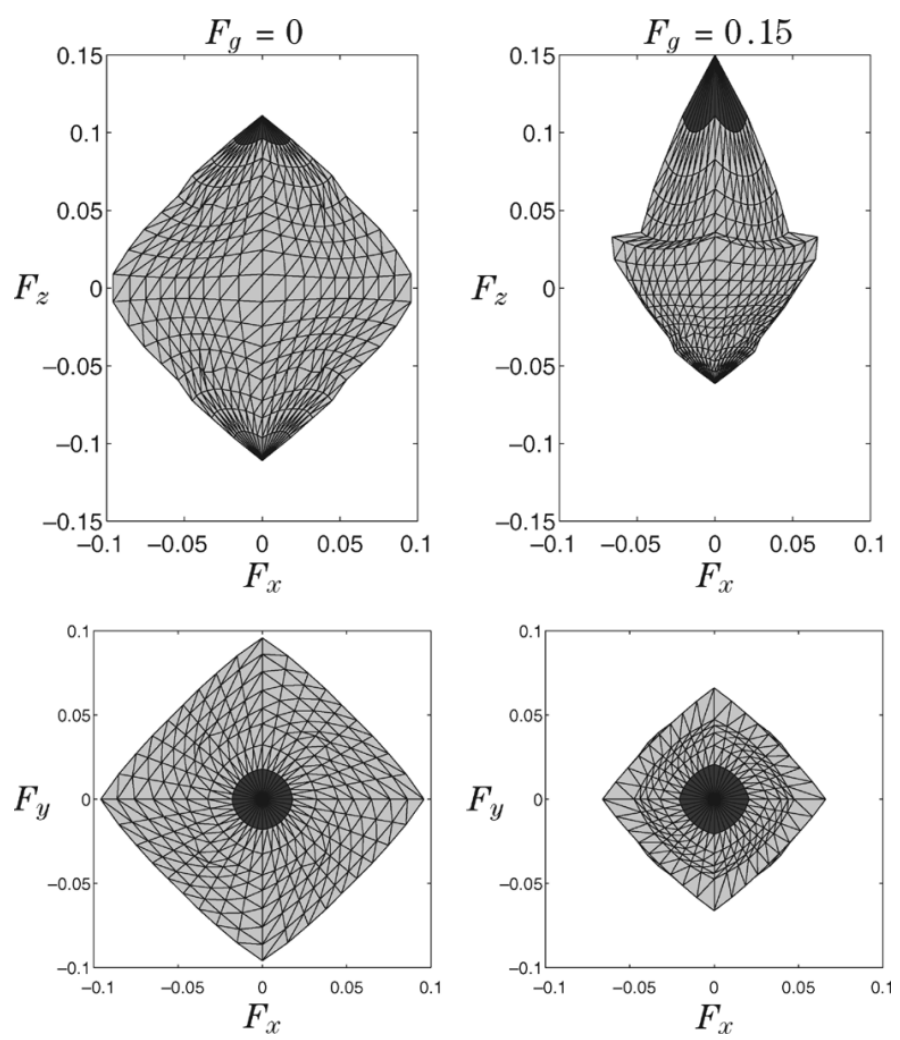

Fig. 6. Illustration of the effect of adding gravity to the grasp in Fig. 5(a). The left-hand figures show two views of the surface $S$ when gravity is neglected. In the right-hand figures, a gravitational force of 0.15 is included. Because $\mu=$ 0.3 , this is also the maximum gravitational force a unit grasp can withstand. Note that the top of $S$ is plotted darker to make the views easier to identify, and that the scale is the same in all the figures.

produce more samples for the force directions near $\theta=0^{\circ}$ and $180^{\circ}$. A uniform, and therefore more efficient, discretization could be made by choosing the force directions as the vertices of a geodesic dome. When evaluating the grasp, the coefficient of friction for the disturbance force was set to 1.5. Note that the friction cone for a vertex can be defined somewhat arbitrarily. Here, it is defined as the union of the cones belonging to the planes forming the vertex. An additional cone, in the direction of the averaged vertex normal, is also added.

Adding Gravity: In the first example, we will look at the effect of adding gravity in the evaluation of a grasp. To simplify interpretation, the grasped object is simply a box and the grasp is symmetrical. The box has dimensions $2 \times 2 \times 5$. The object frame is placed in middle of the box, such that the $z$-axis points along the longer direction of the box. The box is grasped by four frictional point contacts that are symmetrically placed in the $x y$-plane. The coordinates of the contacts are $( \pm 1,0,0)^{\mathrm{T}}$ and $(0, \pm 1,0)^{\mathrm{T}}$; see Fig. 5(a). Solving for the convex hull of the grasp matrix $\mathbf{G}$ results in 416 hyperplanes. The grasp was evaluated two times; first without gravity and then with the gravitational force set to $F_{g}=0.15$. Gravity acts along the negative $z$-axis. Note that $F_{g}=0.3$ is the upper limit for the unit grasp, since it is assumed that $\mu=0.3$. In Fig. 6, two views of $S$ are given for each grasp evaluation. In the first case, $F_{g}=0$, and the problem is totally symmetric. Hence, the surface $S$ is symmetric around all axes, and the grasp has almost equal strength in 

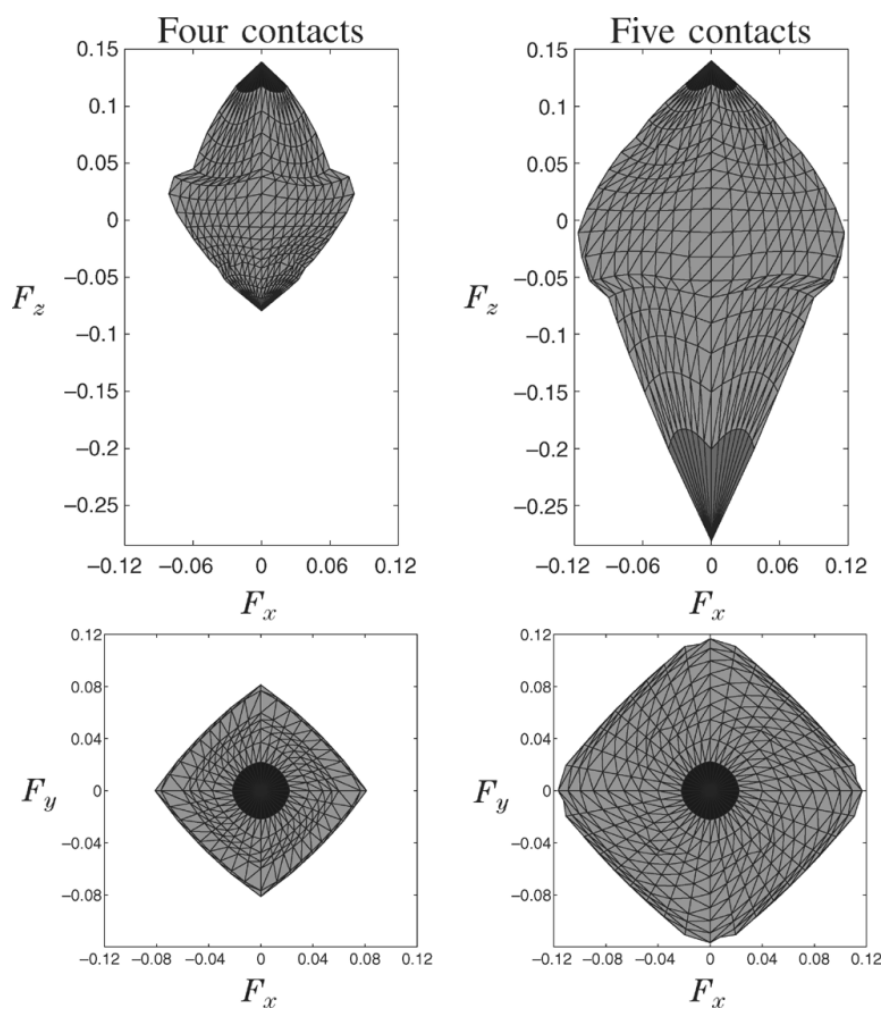

Fig. 7. Left-hand figures show two views of the result for the grasp with four point contacts in Fig. 5(a). Right-hand figures show the result of adding one extra contact, working against the gravity; see Fig. 5(b). The extra contact causes the surface $S$ to expand in all directions, except for the positive $z$-direction. As expected, the expansion is largest in the negative $z$-direction. For both grasps, the gravitational force was 0.1 and the coefficient of friction was 0.3 .

all directions. Breaking the symmetry by adding a (small) gravitational force has the major effect of translating $S$ upwards; compare the upper diagrams in Fig. 6. This is due to upward-directed disturbance forces having to work against the gravitational force, and vice versa. It is also seen that the total volume enclosed by $S$ has decreased due to the grasp being preloaded by a nonzero $\mathbf{W}_{0}$. As the gravitational force is further increased, the volume-reducing effect quickly becomes dominant. This behavior simply reflects the loss of stability margins for the grasp, as it is being used more and more for just holding the weight of the object.

Adding Contacts: In this example, we want to study the effect of adding an additional contact to the grasp in the previous example. In the presence of gravity, the grasp in Fig. 5(a) is weak in the downward direction. To make the most use of an additional contact, it should be placed under the box so that it can work against the gravitational force. The resulting grasp is shown in Fig. 5(b). When comparing the two grasps, the gravitational force was set to 0.1 . The result is shown in Fig. 7. It is seen that the extra contact expands the surface $S$ in all directions, except in the positive $z$-direction. As expected, the expansion is largest in the negative $z$-direction. The expansion of $S$ in the $x y$-plane is explained by the friction force between the box and the extra contact. Due to its large torque arm, this friction force efficiently counteracts the torque generated by a horizontal disturbance applied to the upper or lower parts of the box.
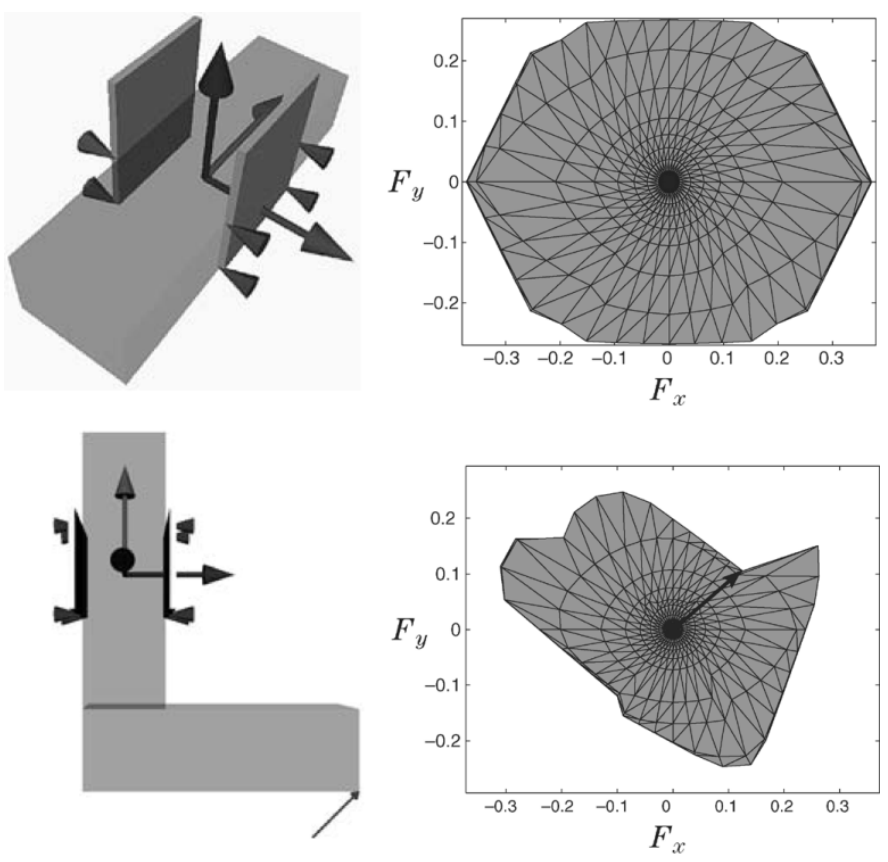

Fig. 8. In the upper-left figure, a box with dimensions $3 \times 10 \times 2$ is grasped with a parallel-jaw gripper, resulting in a total of four point contacts. The upper-right figure shows a view of the resulting surface $S$, assuming no gravity and $\mu=0.3$. In the lower-left figure, the geometry has changed to an L-shape by attaching an identical box to one of the ends. From the lower-right figure, it is seen that the change in geometry has a dramatic effect on the appearance of $S$. In the two lower figures, the worst-case disturbance force in the $x y$-plane is illustrated.

Changing Geometry: As one of the main ideas of the proposed method is to take the object geometry into account, the last example illustrates the effect the geometry can have on the grasp evaluation. The upper-left figure in Fig. 8 shows a box with dimensions $3 \times 10 \times 2$. The box is grasped by a parallel-jaw gripper, resulting in four frictional point contacts. The grasp was evaluated without taking gravity into consideration, and the result is shown in the upper-right figure in Fig. 8. It is seen that the disturbance-force rejection capability is almost identical in all directions in the $x y$-plane. As expected, the grasp is somewhat weaker against forces in the $y$-direction. For such disturbance forces, the grasp can only use the friction forces at each contact to maintain equilibrium.

In the lower-left figure, the same grasp is applied to a different geometry, an L-shaped object composed of two identical boxes. The dimensions are the same as for the previous geometry. A grasp quality measure that only uses the contact points as input would rank these two grasps as equal. It is to be expected, however, that the second grasp has more difficulties rejecting disturbance forces due to the longer torque arms induced by its shape. This is also confirmed by the lower-right figure. In all directions, the disturbance-force rejection capability is significantly reduced, compared with the first grasp. The largest reduction (in the $x y$-plane) occurs at approximately $\pm 45^{\circ}$. This corresponds to a force acting on the lower-right vertex of the geometry, as shown by the arrow. This force is also shown in lower-right figure.

In the lower-right figure in Fig. 8, we can also see some discontinuities in the surface $S$. This can happen when the worstcase disturbance force appears on the boundary of the friction cone for some vertex. A slight change in the direction of the 
force, such that it falls outside the friction cone, will cause the worst case to appear on another vertex. This jump from one vertex to another can be seen as discontinuities in $S$. Note that such discontinuities will not appear for smooth objects.

\section{SUMMARY}

When comparing task-directed and task-independent grasp measures with each other, one most often finds that the former are more complicated and take a longer time to compute. The proposed algorithm breaks this pattern. Task information is easily included by removing object vertices that are unlikely to be in contact during the task, thereby reducing the size of the problem.

One of the main features of the proposed evaluation procedure is that the torque component of the wrench space is used implicitly, thereby reducing the result to a 3-D surface in force space. This surface is easily interpreted and visualized. Furthermore, in the case of a zero-offset wrench, this surface is bounded by a sphere with radius $\sqrt{1+\mu^{2}}$.

An algorithm solving the resulting min-max problem was also proposed. It was shown that introducing a sorting procedure greatly reduced the complexity of the algorithm. Further improvements would involve using more uniformly distributed force directions (e.g., the vertices of a geodesic dome) and downsampling of detailed objects (to reduce the number of vertices that has to be considered).

Offset wrenches, like gravitational forces, are easily taken into account. In some cases, the offset wrench can even have a stabilizing effect on the grasp, as when carrying a plate in the open palm; the weight of the plate helps the grasp to resist horizontal disturbance forces.

The surface $S$ can be used as a quality measure as it is, but in many cases, one would prefer to work with a scalar quality measure. How to derive a scalar quality measure from $S$ is not addressed here, but it is argued that the proposed procedure provides a sound basis for doing so. One suggestion is to choose to choose the minimum $f^{\star}$ over all directions. A force with this magnitude can be resisted by a unit grasp no matter where it is applied and no matter its direction. The drawback of such a quality measure is that it could be overly conservative. If $S$ is a closed surface, one could instead use the volume enclosed by $S$ as a less conservative measure.

The proposed procedure can be used in grasp planning, for finding good grasps for robot grippers. It can also be used as a validation gate in a more reactive manner. If the executed grasp is good enough, the robot will continue its task. Otherwise, the object will be regrasped.

\section{REFERENCES}

[1] A. Bicchi and V. Kumar, "Robotic grasping and contact: A review," in Proc. IEEE Int. Conf. Robot. Autom., San Francisco, CA, Apr. 2000, pp. 348-353.

[2] - "Robotic grasping and manipulation," in Ramsete: Articulated and Mobile Robots for Services and TechnologyS. Nicosia, B. Siciliano, A. Bicchi, and P. Valigi, Eds. Berlin, Germany: Springer-Verlag, 2001, vol. 270, ch. 4, pp. 55-74.

[3] A. Bicchi, "The closure properties of robotic grasping," Int. J. Robot. Res., vol. 14, no. 4, pp. 319-334, Aug. 1995.

[4] R. M. Murray, Z. Li, and S. S. Sastry, A Mathematical Introduction to Robotic Manipulation. Boca Raton, FL: CRC Press, 1994.

[5] A. T. Miller, S. Knoop, H. I. Christensen, and P. K. Allen, "Automatic grasp planning using shape primitives," in Proc. IEEE Int. Conf. Robot. Autom., Taipei, Taiwan, R.O.C., Sep. 2003, vol. 2, pp. 1824-1829.
[6] V.-D. Nguyen, "Constructing force-closure grasps," Int. J. Robot. Res., vol. 7, no. 3, pp. 3-16, Jun. 1988.

[7] C. Ferrari and J. Canny, "Planning optimal grasps," in Proc. IEEE Int. Conf. Robot. Autom., Nice, France, May 1992, vol. 3, pp. 2290-2295.

[8] C. B. Barber, D. P. Dobkin, and H. T. Huhdanpaa, "The Quickhull algorithm for convex hulls," ACM Trans. Math. Softw., vol. 22, no. 4, pp. 469-483, Dec. 1996.

[9] J. Kerr and B. Roth, "Analysis of multifingered hands," Int. J. Robot. Res., vol. 4, no. 4, pp. 3-17, 1986.

[10] J. Ponce and B. Faverjon, "Computing three-finger force-closure grasps of polygonal objects," IEEE Trans. Robot. Autom., vol. 11, no. 6, pp. 868-881, Dec. 1995.

[11] G. M. Bone and Y. Du, "Multi-metric comparison of optimal 2D grasp planning algorithms," in Proc. IEEE Int. Conf. Robot. Autom., Seoul, Korea, May 2001, vol. 3, pp. 3061-3066.

[12] G. Mantriota, "Communication on optimal grip points for contact stability," Int. J. Robot. Res., vol. 18, no. 5, pp. 502-513, May 1999.

[13] D. Kirkpatrick, B. Mishra, and C. K. Yap, "Quantitative Steinitz's theorems with applications to multifingered grasping," in Proc. 20th ACM Symp. Theory Comput., 1990, pp. 341-351.

[14] N. S. Pollard, "Parallel methods for synthesizing whole-hand grasps from generalized prototypes," Ph.D. dissertation, Dept. Elect. Eng. Comput. Sci., Mass. Inst. Technol., Cambridge, MA, 1994.

[15] M. Teichmann, "A grasp metric invariant under rigid motions," in Proc IEEE Int. Conf. Robot. Autom., Minneapolis, MN, Apr. 1996, vol. 3, pp. 2143-2148.

[16] Z. Li and S. S. Sastry, "Task-oriented optimal grasping by multifingered robot hands," IEEE J. Robot. Autom., vol. 4, no. 1, pp. 32-44, Feb. 1988.

[17] B. Mirtich and J. Canny, "Easily computable optimum grasps in 2-D and 3-D," in Proc. IEEE Int. Conf. Robot. Autom., San Diego, CA, May 1994, vol. 1, pp. 739-747.

[18] C. Borst, M. Fischer, and G. Hirzinger, "Grasp planning: How to choose a suitable task wrench space," in Proc. IEEE Int. Conf. Robot. Autom., New Orleans, LA, Apr. 2004, pp. 319-325.

[19] A. T. Miller and P. K. Allen, "Examples of 3D grasp quality computations," in Proc. IEEE Int. Conf. Robot. Autom., Detroit, MI, May 1999, vol. 2, pp. 1240-1246.

[20] W. S. Howard and V. Kumar, "On the stability of grasped objects," IEEE Trans. Robot. Autom., vol. 12, no. 6, pp. 904-917, Dec. 1996.

[21] Q. Lin, J. W. Burdick, and E. Rimon, "A stiffness-based quality measure for compliant grasps and fixtures," IEEE Trans. Robot. Autom. vol. 16, no. 6, pp. 675-688, Dec. 2000.

[22] H. Bruyninckx, S. Demey, and V. Kumar, "Generalized stability of compliant grasps," in Proc. IEEE Int. Conf. Robot. Autom., Leuven, Belgium, May 1998, vol. 3, pp. 2396-2402.

[23] T. H. Cormen, C. E. Leiserson, and R. L. Rivest, Introduction to Algorithms. New York: McGraw-Hill, Jun. 1990.

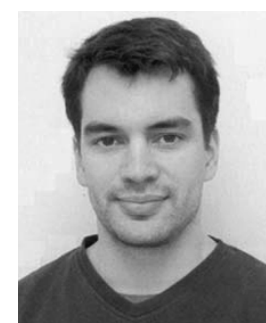

Morten Strandberg was born in Trondheim, Norway, in 1973. He received the M.Sc. and Lic. Eng. degrees in mechanical engineering in 1997 and 2000, respectively, and the Ph.D. degree in automatic control in 2004, all from the Royal Institute of Technology (KTH), Stockholm, Sweden.

In 2004, he joined the Mechatronics and Robot Automation Group, ABB Corporate Research, Västerås, Sweden. His main area of interests are robot path planning, grasping, and robot control.

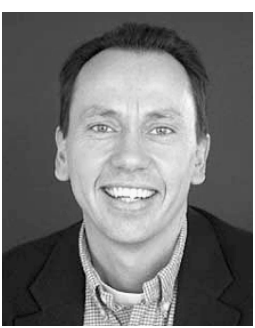

Bo Wahlberg (S'85-M'87-SM'02) was born in Norrköping, Sweden, in 1959. He received the M.Sc. degree in 1983 and the Ph.D. degree in 1987, both from Linköping University, Linköping, Sweden.

In 1991, he became Professor of the Chair of Automatic Control at the Royal Institute of Technology (KTH), Stockholm, Sweden. He was a Visiting Professor at the Information Systems Lab, Stanford University, Stanford, CA, in 1997-1998, and Vice President at KTH from 1999 to 2001 . He is Cofounder of the Centre of Autonomous Systems (CAS) at KTH. His research interests include system identification, modeling and control of industrial processes, and estimation and control of autonomous systems. 